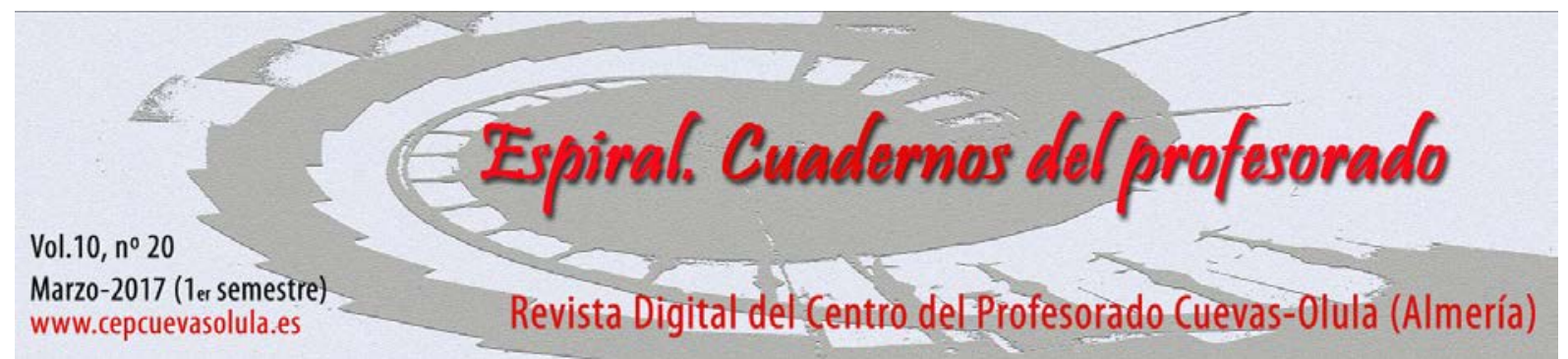

\title{
Experiencias de aprendizaje activo mediante Clickers en prácticas universitarias
}

\section{Active learning experiences using Clickers in university}

\section{Alberto Jesús Perea-Moreno(1), Esther María Salmerón-Manzano(2), Ana María Laguna-Luna $^{(1)}$, María Jesús Aguilera-Ureña ${ }^{(1)}$ y Francisco Manzano-Agugliaro ${ }^{(3)}$}

\author{
${ }^{(1)}$ Departamento de Física Aplicada, Universidad de Córdoba, CeiA3, España \\ ${ }^{(2)}$ Departamento de Derecho, Universidad de Almería, CeiA3, España \\ ${ }^{(3)}$ Departamento de Ingeniería, Universidad de Almería, CeiA3, España
}

RESUMEN: En este trabajo se ha planteado una metodología de aprendizaje proactivo de los estudiantes centrada en el uso de mandos de respuesta interactiva (clickers), complementados con cuestionarios inicial y final. Este método ha sido implantado en las prácticas de laboratorio de Fundamentos Físicos de la Ingeniería de la Universidad de Córdoba, usando tecnología Turning Point ${ }^{\circledR}$, dentro del marco de un proyecto de innovación docente. La evaluación del método se ha realizado de forma cuantitativa mediante una encuesta de satisfacción, contestada por 80 alumnos. Y mediante análisis cualitativo a través de las encuestas de la labor docente del profesorado implicado en este proyecto y las calificaciones medias obtenidas por los alumnos en las prácticas. Se ha obtenido más de un 70\% de satisfacción del método en relación con el aprendizaje: participación en clase, facilidad para expresar su opinión en clase, estar pendiente de las explicaciones, y ayuda para asimilar los conceptos. Los aspectos menos valorados han sido el fomento del trabajo en grupo (51\%) y el aprendizaje de la herramienta (61\%). En resumen, la experiencia ha sido valorada muy positivamente por los alumnos que han sido motivados positivamente y por los profesores a la vez que se les ofrece a estos últimos una herramienta para analizar en cada práctica la asimilación de conceptos previos y los explicados en cada una de ellas.

Palabras clave: Sistema interactivo de respuesta, educación superior, evaluación, prácticas de laboratorio.

ABSTRACT: In this manuscript, a proactive methodology of student learning focused on the use of interactive response controls (clickers), complemented with initial and final questionnaires, has been proposed. This method has been implanted in the laboratory practices for the subjects of Fundamentals of Physics at Engineering degrees of the University of Cordoba, using Turning Point ${ }^{\circledR}$ technology, within the framework of a teacher innovation project. The assessment of the method has been carried out quantitatively through a satisfaction survey, answered by 80 students. And in the other hand, a qualitative analysis through the surveys of the teaching work of the teachers involved in this project and the average qualifications obtained by the students in their practices. More than $70 \%$ satisfaction of the method has been reached in relation to learning: participation in class, express their opinion in class, be aware of the explanations, and understanding of the concepts of the subject. The least valued aspects have been the group work promotion (51\%) and the use of the clickers (61\%). In summary, the experience has been very positively valued by students who have been positively motivated. In summary, the experience has been very well valued by students who have been positively motivated; In regards of teachers, the tool offered in real time the answer of student, so they should able to analyze in each practice the assimilation of previous concepts and those explained in each practice.

Key words: Interactive response system, higher education, assessment, laboratory practice. 
Alberto Jesús Perea-Moreno, A. J., Salmerón-Manzano, E. M.,Laguna-Luna, A. M., AguileraUreña, M. J., y Manzano-Agugliaro, F. (2017). Experiencias de aprendizaje activo mediante Clickers en prácticas universitarias. Espiral. Cuadernos del Profesorado, 10(20), 16-27. Disponible en: http://espiral.cepcuevasolula.es/

Fecha de recepción: 08/10/2016

Fecha de aceptación: 14/01/2017
Enviar correspondencia a: fmanzano@ual.es

\section{Introducción}

La implantación del Espacio Europeo de Educación Superior (EEES) ha introducido reformas importantes sobre las prácticas docentes, y que conllevan al empleo de nuevas metodologías didácticas con objeto de mejorar el proceso de enseñanza-aprendizaje (Ion, Barrera-Corominas \& Tomás-Folch, 2016; Manzano-Agugliaro, 2012). Así, la metodología universitaria no solo debe basarse en la enseñanza sino en un aprendizaje activo, introduciendo tecnologías de la información y de la comunicación (TIC) para alcanzar el objetivo (Delgado \& Oliver, 2006; Guerra, González, \& García, 2010). Tanto es así, que las TIC están recogidas como competencias genéricas en todas las universidades españolas en mayor o menor grado (Manzano-Agugliaro, Salmerón-Manzano, \& PereaMoreno, 2016).

La evolución y desarrollo de las TICs han permitido el desarrollo de nuevas herramientas didácticas en el ámbito de la educación, dando soluciones para acciones formativas a distancia (Manzano-Agugliaro et al., 2016), pero también proporcionando herramientas que dan apoyo a las clases presenciales (Pérez-Romero, Castro-García, León-Bonillo, \& Manzano-Agugliaro, 2016; Castro-García, Pérez-Romero, Leon-Bonillo \& Manzano-Agugliaro, 2017). Es, en este contexto, en el que los sistemas electrónicos de respuesta personal del alumnado se presentan como un elemento tecnológico motivador y una nueva metodología de enseñanza, resultando muy útiles en el aula (Chafer, 2009).

En la investigación en metodologías docentes actual se describen los beneficios de los enfoques de aprendizaje activo (Freeman et al., 2014). Los Clickers, o sistemas de respuesta de estudiantes, son una tecnología utilizada para promover el aprendizaje activo (Agbatogun, 2014). La mayoría de las investigaciones sobre los beneficios del uso de mandos que facilitan la respuesta anónima en el aula han demostrado que los estudiantes se involucran en el aprendizaje y además disfrutan de su uso (Blasco-Arcas et al., 2013).

Las principales ventajas son que los Clickers proporcionan un mecanismo para que los estudiantes participen de forma anónima, y que integran un "enfoque de juego", que pueden involucrar a los estudiantes de una forma más satisfactoria que en una clase tradicional (Stowell \& Nelson, 2007).

Existen diversos estudios previos en los que se demuestran los beneficios de la utilización estos sistemas y sus metodologías, pudiendo destacarse (Bergtrom, 2006; Ausín, Abella, Delgado, \& Hortigüela, 2016; Gómez, 2014): una mejor asimilación del conocimiento sobre el contenido, un método de evaluación proporcionando feedback sobre los resultados, mejora del nivel de atención, así como crear más oportunidades de participación.

Así mismo, los sistemas de respuesta interactiva proporcionan una herramienta de gran valor para potenciar el aprendizaje proactivo, en el que el principal papel del profesor es enseñar a aprender, diseñando actividades de aprendizaje y evaluación, y el alumno es el que construye su propio aprendizaje, realizando las actividades propuestas, pudiendo también autoevaluarse (Dávila, 2000).

Los métodos de enseñanza se pueden clasificar de muchos modos, pero si lo hiciésemos en modo continuo, tendríamos a un extremo la lección magistral en la cual la participación y el control del estudiante es mínimo y en el otro estaría el estudio autónomo donde la intervención del profesor es mínima. Un punto intermedio sería la enseñanza en grupos pequeños generalmente en las carreras de ingeniería o de ciencias esto es el trabajo en el laboratorio. Es en este último modelo, donde existe una 
gran flexibilidad ya que puede estar muy estructurada y controlada rígidamente por el profesor, o puede potenciarse la participación del estudiante donde el profesor puede intervenir más ocasionalmente. Si bien es cierto que los sistemas de respuesta interactivos se han venido usando mayoritariamente en grupos grandes de estudiantes (Banks, 2006), en este trabajo se pretende, extender la nueva metodología basada en el uso de mandos de respuesta interactiva en pequeños grupos o prácticas de laboratorio. Ya que existen experiencias con éxito en este sentido (Borràs et al., 2012), pero en asignaturas de la titulación de química (Fernández \& Pérez, 2013; Fernández, 2014). El origen del planteamiento de esta experiencia radica en intentar mejorar los resultados académicos de los alumnos de las asignaturas de Física de primer curso de Ingeniería en la Universidad de Córdoba, puesto que en los últimos años está siendo anormalmente bajo, de media es superada sólo por el $40 \%$ de los alumnos.

\section{Objetivos}

De acuerdo con lo expuesto, el objetivo principal de esta investigación ha sido el diseño, implementación y análisis de una nueva metodología docente basada en el uso de los sistemas de respuesta interactiva (Clikers) en las prácticas de laboratorio de las disciplinas de Fundamentos Físicos de la Ingeniería. Para la consecución de este objetivo, es necesario plantear los siguientes objetivos específicos:

- Diseño de una metodología docente para el desarrollo de sesiones prácticas de laboratorio, que incluya la utilización de mandos interactivos de respuesta (Clikers).

- Análisis de la utilidad de la nueva metodología docente.

\section{Material y Métodos}

\section{Material}

Esta investigación se enmarca dentro de una línea de innovación docente iniciada por miembros del Departamento de Física Aplicada de la Universidad de Córdoba en los últimos cursos, con el uso de los sistemas de respuesta interactiva como nueva metodología docente para la enseñanza de la Física en los estudios de Ingeniería. Financiado con el anterior proyecto, y cofinanciado por el Departamento de Física Aplicada, se disponía de un sistema Turning Point ( (comercializado por la empresa Turning Technologies). En este proyecto se diseñó una nueva metodología docente basada en el uso de los Sistemas de Respuesta Interactiva para su aplicación y validación en la

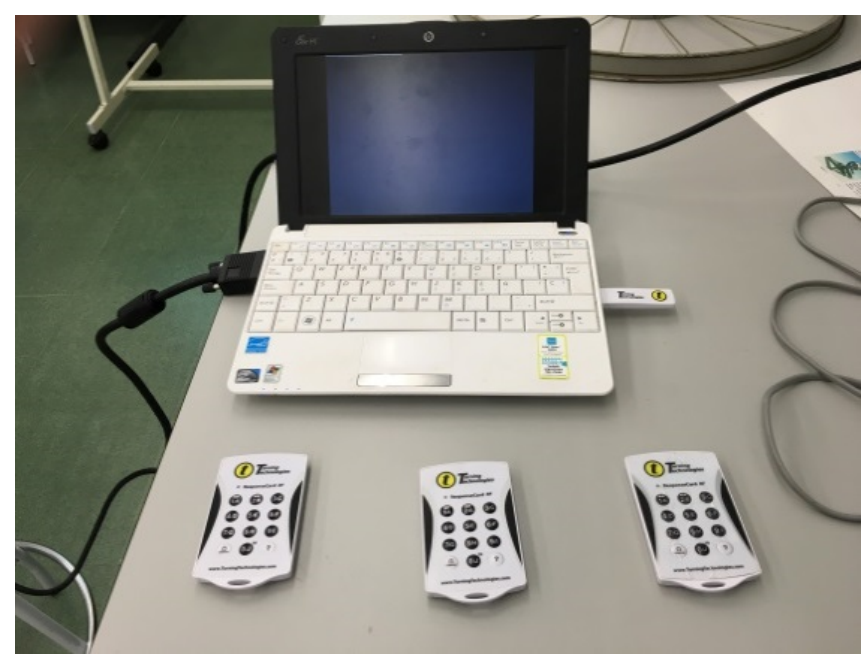

Figura 1. Equipo utilizado: portátil, tarjeta receptora y mandos de respuesta. enseñanza de la Física en los Grados en Ingeniería que se imparten en la Universidad de Córdoba.

El sistema consta de una tarjeta receptora con conexión USB al ordenador del profesor, y un conjunto de mandos de respuesta que se reparten a los alumnos (Figura 1). Cada pregunta debe tener un propósito pedagógico específico, referido a un concepto, a un procedimiento o a un objetivo metacognitivo (Beatty et al., 2006). Mediante presentaciones PowerPoint, el profesor puede realizar cuestionarios (verdadero/falso, opción múltiple...) a los alumnos presentes en el aula. Las respuestas emitidas por los alumnos a través de su mando de respuesta, son recogidas con el software adecuado, que gestiona toda la información (Figura 2). 


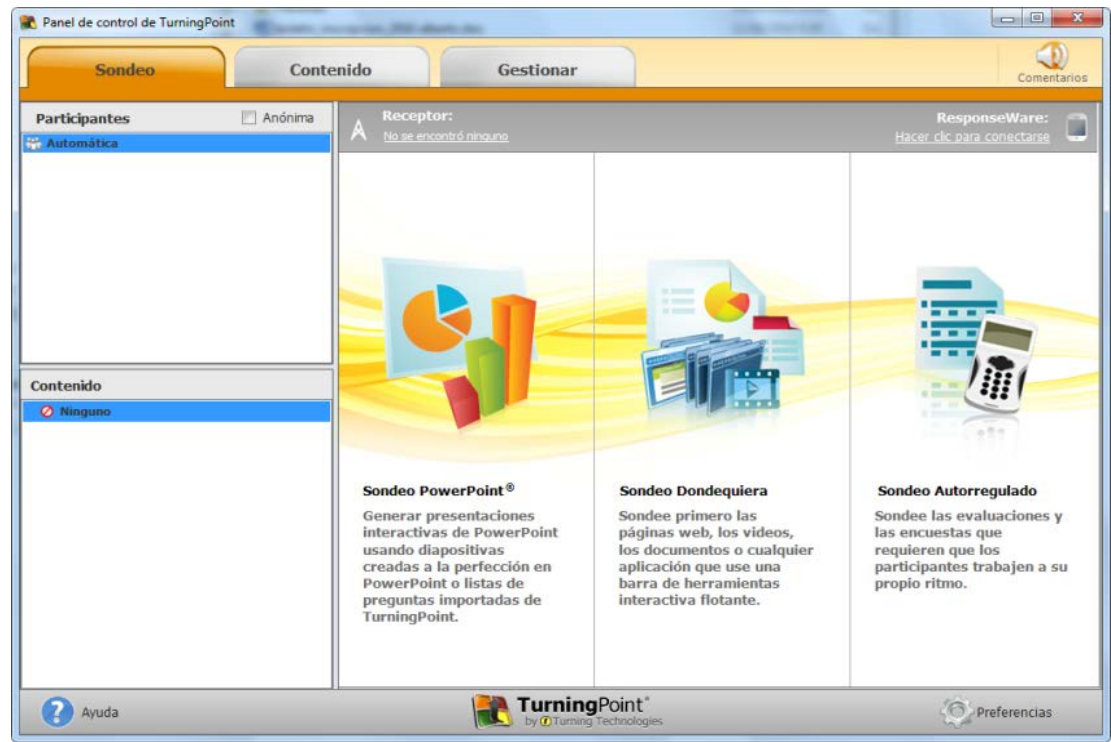

Figura 2. Software Turning Point.

\section{Metodología}

La metodología planteada es la de la Figura 3, donde existen dos fases, una de trabajo previo en casa y otra ya en el aula.

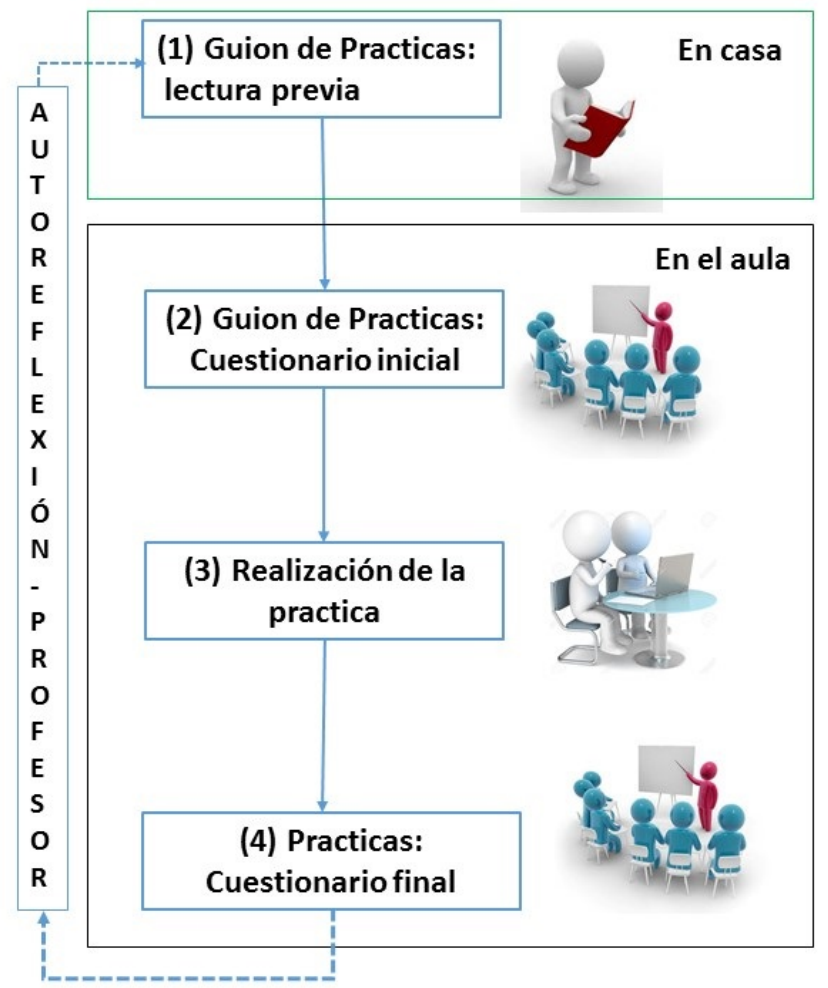

Figura 3. Metodología.

\section{Preparación previa de la práctica por los alumnos}

En la metodología propuesta en las prácticas de laboratorio de Fundamentos Físicos de la Ingeniería, el alumno tiene a disposición un guion de la práctica, que debe haber leído y entendido antes de asistir a la sesión de prácticas correspondiente. 


\section{Cuestionario Inicial}

Al comienzo de la sesión práctica, el profesor plantea un cuestionario inicial, usando el SW Turning Point. En la Figura 4 se ilustra una sesión práctica con el sistema expuesto. Una vez presentada cada diapositiva con la cuestión planteada, los alumnos disponen de un tiempo para emitir su respuesta con los clickers. Pasado el tiempo, se muestra en pantalla la respuesta verdadera, así como el porcentaje de alumnos en base a cada una de las respuestas seleccionadas.

En la Figura 5 se muestran varias imágenes del cuestionario previo. Estos cuestionarios son presentaciones de Power-Point, de forma que se permite incluir en ellas figuras aclaratorias para el alumno. Se observa como la primera diapositiva muestra los objetivos de la práctica. Cada diapositiva supone una pregunta del cuestionario previo. En la figura se muestran ejemplos de preguntas (diapositivas) del cuestionario previo una vez contestadas por los alumnos. La respuesta correcta se ve enmarcada y los porcentajes de cada respuesta de los alumnos aparecen a la derecha de la

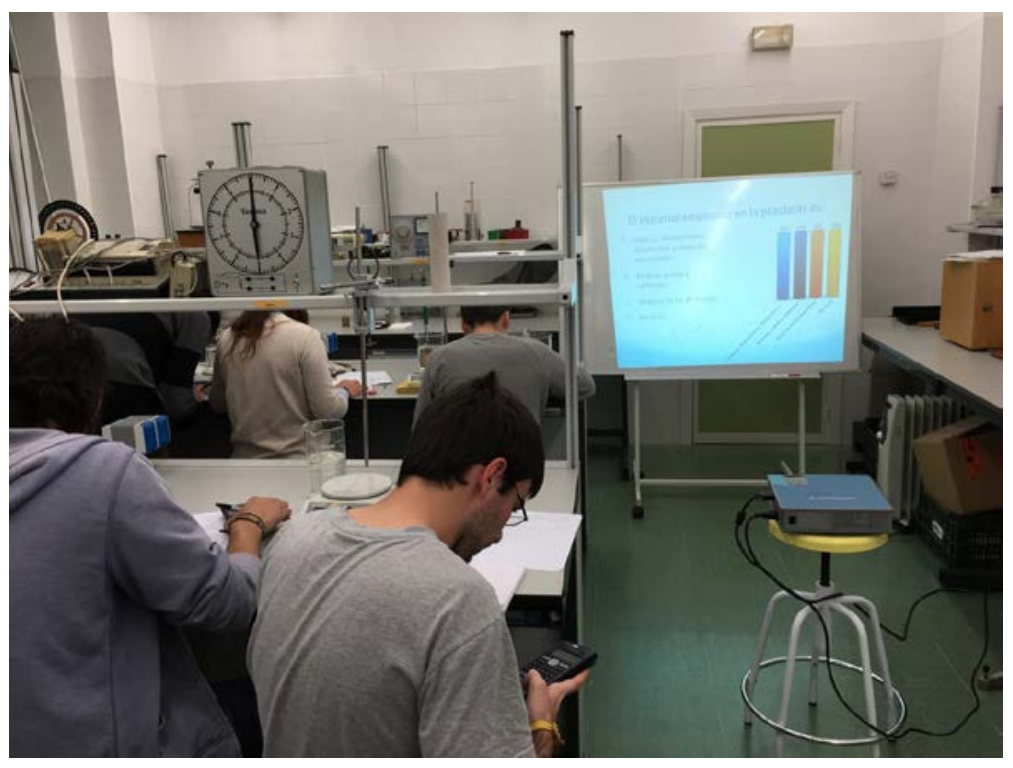

Figura 4. Ejemplo de sesión práctica en laboratorio.

diapositiva. Los alumnos disponen de un tiempo de alrededor de 2 minutos para contestar cada pregunta, aunque se ha comprobado que no se agota nunca ese tiempo.

\section{Cuestionario Final}

Una vez que los alumnos han terminado el trabajo en el laboratorio siguiendo el guion de la práctica, se les plantea otro cuestionario con el que se evalúa el nivel de aprovechamiento de la sesión y de asimilación de las competencias objeto de la práctica. De manera similar al cuestionario inicial, pasado el tiempo para contestar cada cuestión, el alumno ve la respuesta correcta, lo que le permite su autoevaluación. Así mismo, a la vista de los resultados, el profesor puede dar las explicaciones que considere oportunas. En la Figura 6 se ilustra un ejemplo de cuestionario final todavía sin contestar por los alumnos. Obsérvese que es un cuestionario más complejo que el inicial, donde solo se preguntaban conceptos previos necesarios, en este se pretende conocer el grado de asimilación de la práctica y asimilación de los conceptos de la misma.

El cuestionario que se pasa a los alumnos a la finalización de la sesión permite asignar una calificación objetiva sobre el nivel de aprovechamiento de cada uno. Los alumnos tienen el mismo tiempo para contestar este cuestionario que en el cuestionario inicial, también se observó que el tiempo no es un factor limitante. El sistema permite identificar cada respuesta del alumno a través del código del mando que está usando, y esta información se almacena en una base de datos que el profesor puede utilizar posteriormente para su análisis, quedando la información almacenada por tanto de forma anónima. La Figura 7 muestra el resultado en Excel guardado para la práctica ejemplo seleccionada, sesión del 03-05-2016 realizada a las 16:28 horas por 8 alumnos, obteniendo una puntuación promedio de 71,88\%. Puede observarse como se da la calificación por alumno (según el código del mando) por filas, y por pregunta realizada (columnas). El color rojo indica que no se ha acertado la respuesta (la cual viene en la segunda fila, clave de respuesta), mientras que el color verde indica que la respuesta es correcta (coincide con la de la segunda fila, clave de respuesta). Así, a simple vista se observa como 
hay conceptos que no han sido entendidos por los alumnos, por ejemplo, la primera pregunta, y otras que son entendidas por todos, como es el caso de las preguntas 6, 7,10 y 11.

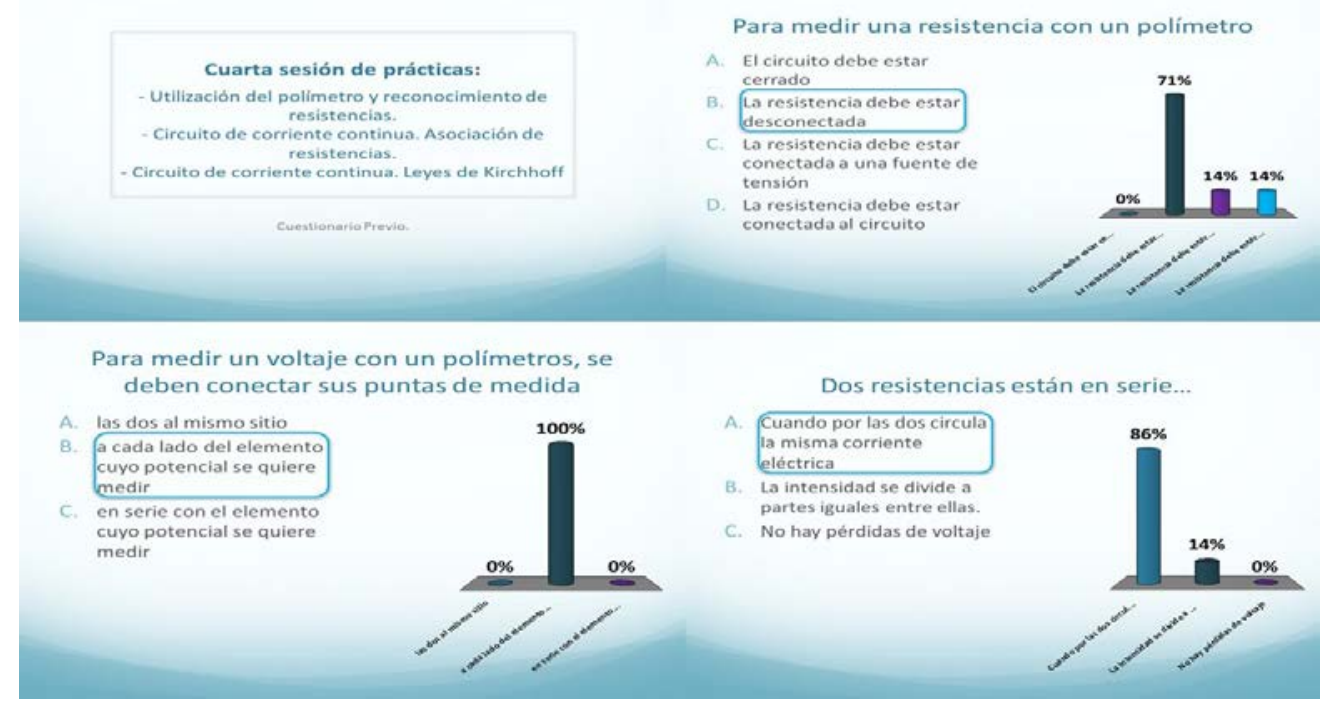

Figura 5. Ejemplo de cuestiones del cuestionario previo en modo presentación Power Point.

Por otro lado, con las respuestas de los alumnos, se puede plantear una autorreflexión de la docencia por parte del profesor de los contenidos y explicación de la práctica, véase Figura 3. Se puede considerar como un elemento de autoevaluación del profesor responsable de la práctica, tan necesarios en la universidad actual (Chavarría, 2016). Esto se puede considerar como una retroalimentación del sistema para el diseño de las prácticas.

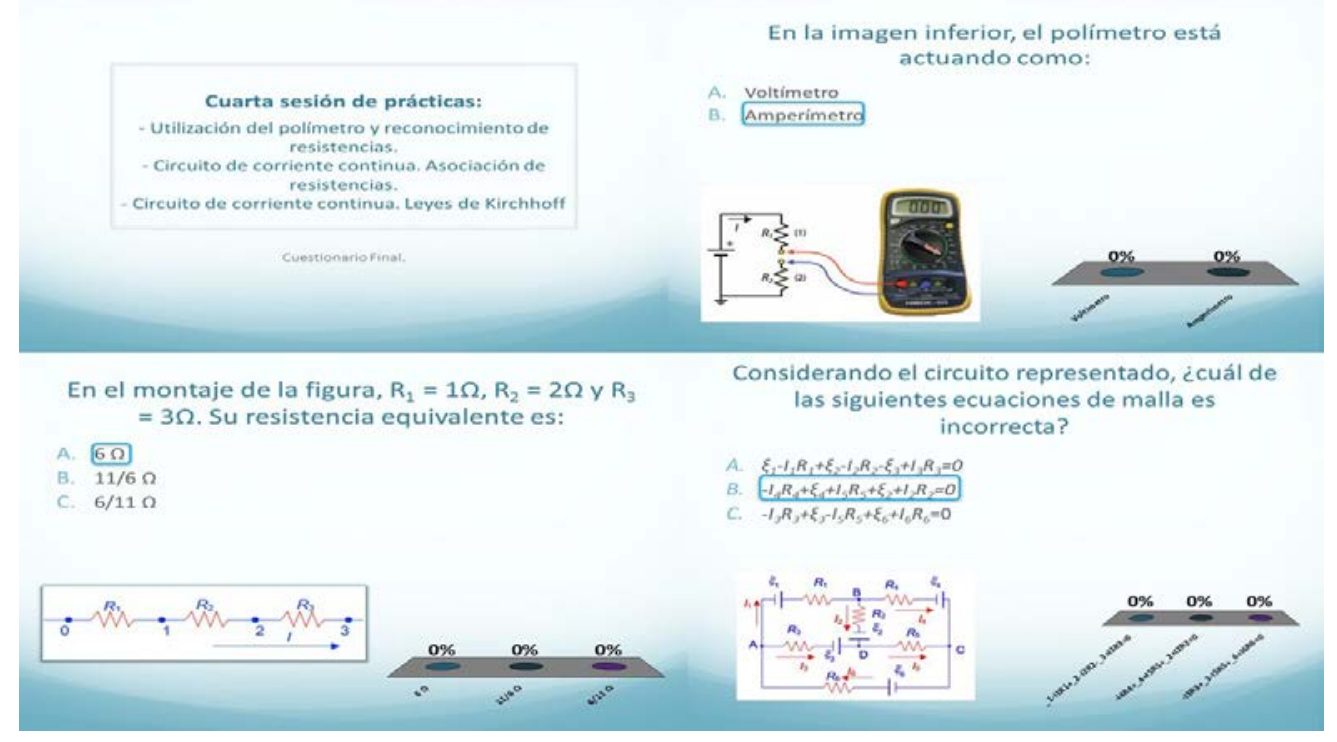

Figura 6. Ejemplo de plantilla de diapositivas de cuestionario final, que emplea el SW Turning Point, antes de ser contestada por los alumnos.

\section{Evaluación de la Experiencia}

La metodología presentada debe ser evaluada para poder ser validada, por ello se recurrió a valorar los siguientes aspectos:

a) Encuesta de satisfacción del alumnado. Esta encuesta se realizó mediante un link a un formulario de google (google forms). 
b) Asistencia a clase: Aumento o disminución.

c) Valoración del profesorado en las encuestas docentes.

La Tabla 1 recoge las preguntas de la encuesta realizada a los alumnos. En dicha encuesta se pide a los alumnos que muestren su grado de acuerdo/desacuerdo con diferentes preguntas sobre el uso del sistema de respuesta interactiva en las prácticas de laboratorio, utilizando una escala Likert 1 (total desacuerdo) - 5 (acuerdo total).

\begin{tabular}{|c|c|c|c|c|c|c|c|c|c|c|c|c|c|c|}
\hline $\begin{array}{l}\text { ID de } \\
\text { dispositivo }\end{array}$ & P1 & P2 & P3 & P4 & P5 & P6 & P7 & P8 & P9 & P10 & P11 & P12 & $\begin{array}{l}\text { Total de } \\
\text { puntos }\end{array}$ & Puntuación \\
\hline $\begin{array}{l}\text { Clave de } \\
\text { respuesta }\end{array}$ & A & A & B & B & B & D & B & A & A & A & B & A & 12,00 & $100,00 \%$ \\
\hline 3DD981 & B & A & c & B & c & D & B & B & c & A & B & A & 7,00 & $58,33 \%$ \\
\hline $3 F 5085$ & B & A & B & B & B & D & B & A & A & A & B & A & 11,00 & $91,67 \%$ \\
\hline $3 \mathrm{~F} 5020$ & B & A & B & B & B & D & B & A & A & A & B & - & 10,00 & $83,33 \%$ \\
\hline $3 \mathrm{~F} 505 \mathrm{~B}$ & c & A & c & c & B & D & B & A & A & A & B & A & 9,00 & $75,00 \%$ \\
\hline $3 \mathrm{~F} 50 \mathrm{D} 4$ & B & B & D & c & B & D & B & A & A & A & B & A & 8,00 & $66,67 \%$ \\
\hline $3 F 5062$ & c & D & D & c & c & D & B & A & A & A & B & A & 7,00 & $58,33 \%$ \\
\hline $3 \mathrm{~F} 50 \mathrm{CB}$ & c & B & D & B & c & D & B & A & A & A & B & A & 8,00 & $66,67 \%$ \\
\hline $3 \mathrm{~F} 50 \mathrm{ED}$ & B & A & D & B & B & D & B & A & A & A & B & - & 9,00 & $75,00 \%$ \\
\hline $\begin{array}{l}\text { Promedios de } \\
\text { listas de } \\
\text { participantes }\end{array}$ & $0,00 \%$ & $62,50 \%$ & $25,00 \%$ & $62,50 \%$ & $62,50 \%$ & $100,00 \%$ & $100,00 \%$ & $87,50 \%$ & $87,50 \%$ & $100,00 \%$ & $100,00 \%$ & $75,00 \%$ & $862,50 \%$ & $71,88 \%$ \\
\hline
\end{tabular}

Figura 7. Ejemplo de respuestas finales almacenadas en formato Excel para el cuestionario de la práctica ejemplo (doce preguntas).

\section{Tabla 1}

Preguntas de la encuesta de satisfacción del alumnado

\begin{tabular}{ll}
\hline $\mathbf{N}^{\mathbf{0}}$ & \multicolumn{1}{c}{ Pregunta } \\
\hline 1. & Con el uso de los mandos aumenta mi nivel de participación en clase \\
2. & Con el uso de los mandos me resulta más fácil expresar mi opinión en clase \\
3. & Con el uso de los mandos, estoy más pendiente de las explicaciones para responder \\
correctamente a las preguntas planteadas & El hecho de conocer si mi respuesta es correcta de manera inmediata, me facilita asimilar los \\
5. & Conceptos expuestos en clase \\
6. & El uso de los mandos interactivos fomenta el trabajo en grupo \\
7. & Es fácil aprender el funcionamiento de los mandos y saber si los estoy usando bien \\
8. & El uso de los mandos no entorpece el desarrollo de las clases \\
9. & Me parece una actividad académica interesante y útil \\
10. & A continuación, te pedimos que hagas una valoración global del recurso educativo
\end{tabular}




\section{Resultados}

En la Figura 7, a simple vista se observa como hay conceptos que no han sido entendidos por los alumnos, por ejemplo, la primera fila esta respondida correctamente por $0 \%$ de los alumnos, mientras que otras son entendidas por todos (100\%) como las preguntas 6, 7,10 y 11 . Por tanto, el análisis de los resultados de los cuestionarios inicial y final permite al profesor conocer el grado de asimilación de los conceptos por parte de los alumnos, corrigiendo por retroalimentación los errores de partida.

La Tabla 2 recoge los resultados de la encuesta de satisfacción, donde se han descrito también en tanto por ciento las respuestas de 1 a 5 de los 80 alumnos que han contestado a la encuesta. La media ponderada hace referencia al valor medio de la encuesta expresado en tanto por ciento, siendo el valor 5 el $100 \%$. Se observa como todas las respuestas tienen un alto grado de aceptación en promedio por encima del $70 \%$. La pregunta peor valorada es si el uso de estos dispositivos fomenta el trabajo en grupo, la cual está al 50\%. La segunda cuestión peor valorada, por debajo de la media es la facilidad para el uso de la herramienta con el $61 \%$. El aspecto más positivo, y por tanto más valorado ha sido el conocer si la respuesta es correcta de manera inmediata facilita asimilar los conceptos expuestos en clase, con el $82.5 \%$ de satisfacción. Por otro lado, el segundo aspecto más valorado ha sido que con el uso de los mandos les resulta más fácil expresar su opinión en clase, con más del $77 \%$ de los encuestados a favor, y esto era unos de los objetivos del desarrollo de la experiencia docente.

Tabla 2

Resultados de las encuestas de satisfacción (preguntas Tabla 1)

\begin{tabular}{|c|c|c|c|c|c|c|c|c|c|c|c|c|}
\hline \multirow[b]{2}{*}{ Pregunta } & \multicolumn{2}{|c|}{ Valor 5} & \multicolumn{2}{|c|}{ Valor 4} & \multicolumn{2}{|c|}{ Valor 3} & \multicolumn{2}{|c|}{ Valor 2} & \multicolumn{2}{|c|}{ Valor 1} & \multicolumn{2}{|c|}{ Media } \\
\hline & n & $\%$ & n & $\%$ & $\mathrm{n}$ & $\%$ & $\mathrm{n}$ & $\%$ & n & $\%$ & n & $\%$ \\
\hline 1 & 13 & 16.25 & 36 & 45.00 & 28 & 35.00 & 3 & 3.75 & 0 & 0.00 & 3.74 & 74.75 \\
\hline 2 & 25 & 31.25 & 23 & 28.75 & 28 & 35.00 & 4 & .000 & 0 & 0.00 & 3.86 & 77. \\
\hline 3 & 10 & 12.50 & 35 & 43.75 & 28 & 35.00 & 4 & 5.00 & 3 & 3.75 & 3.56 & 71. \\
\hline 4 & 35 & 43.75 & 28 & 35.00 & 11 & 13.75 & 4 & 5.00 & 2 & 2.50 & 4.13 & 82.50 \\
\hline 5 & 13 & 16.25 & 35 & 43.75 & 28 & 35.00 & 4 & 5.00 & 0 & 0.00 & 3.71 & 74. \\
\hline 6 & 4 & 5.00 & 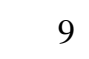 & 1 & 28 & 3 & 28 & 35.00 & 11 & 13.75 & 2.59 & 51. \\
\hline 7 & 11 & 13 & 20 & 25 & 20 & 25.00 & 20 & 25.00 & 9 & 11.25 & 3.05 & 61.00 \\
\hline 8 & 13 & 16.25 & 37 & 46.25 & 30 & 37.50 & 0 & 0.00 & 0 & 0.00 & 3.79 & 75.75 \\
\hline 9 & 12 & 15.00 & 35 & 43.75 & 28 & 35.00 & 4 & 500 & 1 & 1.25 & 3.66 & 732 \\
\hline 10 & 12 & .00 & 34 & 42. & 29 & 36.2 & 3 & -7 & 2 & 2.50 & 3.64 & 72 \\
\hline Media & 14.8 & 18.50 & 29.2 & 36.50 & 25.8 & 32.25 & 7.4 & 9.25 & 2.8 & 3.50 & 3.6 & 71.45 \\
\hline
\end{tabular}

Dado que la asistencia a prácticas es obligatoria no se ha observado incremento en la asistencia a las mismas. Pero si se ha notado el aumento de la asistencia en las clases de teoría, pasando del 50\% en años anteriores al 75\% desde la implantación de esta metodología en prácticas. Este hecho se atribuye a intentar comprender mejor los guiones de prácticas.

Respecto a las encuestas de la labor docente del profesorado se ha observado que un incremento de 0.7 puntos de media en dicha encuesta. Aunque hay que ser prudentes con este dato, pues puede deberse a muchos factores, pero en cualquier caso hay que entenderlo como un dato positivo. 


\section{Discusión}

La aplicación de la metodología propuesta ha dado resultados de satisfacción recogida en la encuesta realizada por encima del $70 \%$ en los siguientes aspectos relacionados con el aprendizaje: participación en clase, facilidad para expresar su opinión en clase, estar pendiente de las explicaciones, y ayuda para asimilar los conceptos. Respecto a la herramienta en sí, hay también satisfacción por encima del 70\% para: que la respuesta sea anónima. El anonimato no permitirá el uso de las respuestas para la evaluación de los estudiantes (Lantz, 2010). En nuestro caso, se gana con el beneficio del anonimato inmediato en clase para aumentar la participación y la implicación con las prácticas, pero el conocer la respuesta de cada alumno puede servir para una futura evaluación del mismo. En definitiva, se ha comprobado que el alumno adopta una actitud más activa, y a la vez puede servir de autoevaluación para el alumno. Se prevé que en un futuro se pueda puntuar hasta el $50 \%$ de la nota de prácticas con el resultado de las respuestas de los alumnos a los cuestionarios finales. Esto aumentaría toda más la motivación por las prácticas.

Las prácticas se hacen en grupos de tres personas (pues no se dispone de material para hacerlas individualmente) pero las respuestas a las preguntas sí que se responden de forma individual para asegurar que cada alumno ha asimilado el concepto de la práctica. Esto puede ser el motivo que los peores resultados se observan para el fomento del trabajo en grupo (51\%). Este aspecto sin duda debe plantear una reflexión para los cursos siguientes, buscar alguna forma de fomentar este aspecto, pero sin perder de vista que algún alumno se puede escudar en el grupo para simplemente asistir a la práctica sin realizar aprovechamiento. Quizás la solución podría venir por plantear preguntas al grupo que deben ser consensuadas para fomentar esta competencia.

El aprendizaje del uso del mando no es tan intuitivo como hubiese sido deseable, pues la satisfacción está al 61\%. Entendemos que esto es fruto de la incorporación de una nueva tecnología en el aula, si esta experiencia fuese más extendida probablemente se vería más intuitiva. En general, estamos de acuerdo en que "la creencia del profesor sobre el uso de la tecnología en el aula influye en su percepción hacia las mejoras a nivel de aula” y en concreto: comunicación profesor-alumno, clima de aula, participación de los alumnos y trabajo autónomo (Boza et al., 2010), y por tanto sobre su implantación. No obstante, se entiende que se está trabajando también la competencia transversal de uso de las TIC (Tecnologías de la Información y las Comunicaciones) que es una competencia muy extendida en las universidades andaluzas (Manzano-Agugliaro et al., 2016b).

En cuanto a la precepción de la satisfacción global del alumnado, los datos son los muy buenos ya que entienden que no empeoran el aprendizaje, les parece interesante y útil, y valoran globalmente el recurso educativo como bueno por encima del 70\%. Sin embargo, hay cuestiones que debemos reflejar o plantear respecto de la metodología propuesta. El primer aspecto seria cuánto tiempo emplea el alumno en prepararse/leer la práctica en casa. Este aspecto no se ha evaluado cuantitativamente, pero cada guion de prácticas suele tener 10 páginas por lo que se estima que el estudiante debería emplear 20 minutos en leer y comprender la práctica. Se ha observado, que en años anteriores ningún alumno, prácticamente sin excepción, había leído el guion de prácticas y menos aún repasado la clase de teoría pasa asistir a una sesión de prácticas. El hecho de existir un cuestionario inicial, ha conseguido revertir la situación, que todos los alumnos sin excepción lean la práctica que van a realizar, y muchos repasen los contenidos de teoría para entender lo que van a realizar. De hecho, las notas de prácticas han mejorado de media en un $10 \%$ y dado que las prácticas tienen porcentaje sobre la nota final de la asignatura, se puede decir que hay una contribución positiva de forma numérica. Además, se considera que en cierto modo se está generando un cierto hábito de estudiar la asignatura, aunque sea de manera semanal para asistir a la sesión de prácticas, esto es aumentar el trabajo autónomo real del alumno, pero no así el volumen teórico del mismo para superar la asignatura.

No debemos olvidar el recurso del tiempo que se emplea en los cuestionarios durante las sesiones de prácticas, puesto que podría parecer que esta merma el tiempo que se les dedica. En promedio se dedica 10 minutos al cuestionario inicial y lo mismo para el final (12 preguntas) cuando las sesiones son de 2 horas, y de 5 minutos cuando la sesión es de una hora (6 preguntas). Esto supone 
un $15 \%$ del tiempo de la práctica. Pero se ve ampliamente compensada por el tiempo que se ahorra en la explicación de la práctica, puesto que viene leída de casa. Esto no sucedía así en el modo tradicional, donde el profesor comprobaba que raramente lo habían estudiado, con lo que debía dedicar mucho tiempo de la sesión a explicar los fundamentos y metodología de las prácticas. Con la nueva metodología, el hecho de que los alumnos saben que al inicio de la práctica deben hacer un cuestionario sobre contenidos básicos presentados en el guion de la práctica, hace que acudan a la práctica más preparados, lo que les lleva a tomar una actitud más activa. De otro modo habría que invertir tiempo en la explicación en el aula, al menos en esa cantidad. Luego no se considera que exista pérdida de tiempo. Todo ello redunda en un mejor aprovechamiento del tiempo en laboratorio, y mejor asimilación de los contenidos de la práctica, tal como se comprueba de la mejora de las calificaciones medias de prácticas y de los resultados de los cuestionarios finales de cada práctica. En resumen, la metodología empleada permite un mejor aprovechamiento del tiempo en laboratorio, que es cada vez más limitado por el sistema de créditos, que hace imposible repetir una práctica.

Desde el punto de vista de los profesores participantes en el proyecto, han sido mejor valorados que el año anterior en la encuesta docente, si bien no se puede atribuir este hecho a la simple adopción de este método, puede que su actitud se vea más motivada en general o que sea la percepción del alumnado, o simplemente el sacar mejores calificaciones de prácticas les haga valorar mejor el profesorado. Por otro lado, el profesor cuenta con una herramienta que permite la realimentación, explicando conceptos mal entendidos por los alumnos al inicio de la sesión, por ejemplo, conceptos que no han sido entendidos por un grupo de prácticas anterior, el profesor puede hacer hincapié en los mismos, véase por ejemplo la pregunta 1 de la practica 4 en la Figura 6, no contestada por ningún alumno. Esto indica que la pregunta debe ser reformulada, el concepto aclarado en teoría o mejor explicado en el guion de prácticas.

Los resultados del proyecto en general fueron muy satisfactorios por parte de los discentes. Se ha llegado a la conclusión de que la tecnología ha tenido una buena aceptación por parte de los alumnos, motivándoles a realizar un buen trabajo en laboratorio. Y por tanto se ha comprobado que los sistemas de respuesta interactiva son especialmente útiles en una primera etapa del proceso de enseñanza/aprendizaje para detectar los conceptos previos erróneos que han adquirido los alumnos y, a partir de su identificación, proponer las actividades docentes adecuadas para la corrección de los mismos y favorecer el aprendizaje (López-Quintero, Varo-Martínez, Laguna-Luna, \& Pontes-Pedrajas, 2016). Asimismo, son útiles para medir, durante el desarrollo de los contenidos teóricos, el grado de asimilación de los mismos por parte del alumnado, detectando en el mismo momento de la acción docente las dificultades que presentan los estudiantes así como los conceptos que no se han entendido adecuadamente y, de esta forma, poder tomar las medidas oportunas para la corrección de los mismos.

Con todo lo expuesto anteriormente, esta experiencia se ha demostrado la utilidad de los mandos de respuesta interactiva en las prácticas de laboratorio de Fundamentos Físicos de la Ingeniería, y creemos que debería ser extensible a otras áreas de conocimiento y otras titulaciones, para mejorar el aspecto de familiarizarse con el funcionamiento de los mandos.

\section{Conclusiones}

A la vista de los resultados obtenidos, se puede concluir que la satisfacción de los estudiantes está por encima del $70 \%$ en los aspectos relacionados con el aprendizaje como son la participación en clase, motivación, y asimilación de contenidos. También se ha visto que debe mejorarse el aspecto de trabajo en equipo, pues el uso de mandos provoca quizás un individualismo, ya que los resultados muestran que sólo la mitad del alumnado está de acuerdo con que se fomenta esta competencia. Los profesores participantes en el proyecto opinan que sería muy recomendable extender esta experiencia a más asignaturas de las titulaciones universitarias para amortiguar la relativa baja satisfacción con la facilidad de aprendizaje del manejo de los clickers (61\%). Para aumentar la motivación del estudiante por las prácticas, se prevé que en un futuro se pueda puntuar hasta el $50 \%$ de la nota de prácticas con el resultado de las respuestas de los alumnos a los cuestionarios finales. En conclusión, al darles a los estudiantes una retroalimentación inmediata sobre lo aprendido, se observa que los alumnos consiguen 
motivarse y que estos se involucren activamente en el proceso de aprendizaje, creando objetivos a corto plazo lo que les hace necesariamente estar más atentos a la explicación del docente y llevar la asignatura más al día de lo que lo hacían sus compañeros de años anteriores.

\section{Referencias}

Agbatogun, A. O. (2014). Developing learners' second language communicative competence through active learning: clickers or communicative approach?. Educational Technology \& Society, 17(2), 257-269.

Ausín, V., Abella, V., Delgado, V., \& Hortigüela, D. (2016). Project-based learning through ICT. an experience of teaching innovation from university classrooms. [Aprendizaje basado en proyectos a través de las TIC. Una experiencia de innovación docente desde las aulas universitarias] Formación Universitaria, 9(3), 31-38. doi:10.4067/S0718-50062016000300005.

Banks, D. A. (2006). Reflections on the use of ARS with small groups. Audience response systems in higher education, 373-386.

Beatty, I. D., Gerace, W. J., Leonard, W. J., \& Dufresne, R. J. (2006). Designing effective questions for classroom response system teaching. American Journal of Physics, 74(1), 31-39.

Bergtrom, G. (2006). Clicker sets as learning objects. Interdisciplinary journal of knowledge and learning objects, 2(1), 105-110.

Blasco-Arcas, L., Buil, I., Hernández-Ortega, B., \& Sese, F. J. (2013). Using clickers in class. The role of interactivity, active collaborative learning and engagement in learning performance. Computers \& Education, 62, 102-110.

Borràs, J. F., Valls, A. I., Guio, J. R. T., Olate, G. U., Minguez, J. B., Carrasco, G. V., \& Costas, T. P. (2012). Uso de sistemas de respuesta inmediata (clickers) para evaluar las prácticas de laboratorio: mejora del aprendizaje de los alumnos y de la enseñanza de los profesores. Revista del Congrés Internacional de Docència Universitària i Innovació (CIDUI), 1(1).

Boza, A; Tirado, R. \& Guzmán-Franco, M-D (2010). Creencias del profesorado sobre el significado de la tecnología en la enseñanza: influencia para su inserción en los centros docentes andaluces. RELIEVE, 16(1), 1-24. Disponible en: http://www.uv.es/RELIEVE/v16n1/RELIEVEv16n1_5.htm

Castro-García, M., Pérez-Romero, A. M., León-Bonillo, M. J., \& Manzano-Agugliaro, F. (2017). Developing Topographic Surveying Software to Train Civil Engineers. Journal of Professional Issues in Engineering Education and Practice, 143(1), 04016013

Chafer, E. (2009). Una introducción a los sistemas de respuesta interactiva. Electrónica y Comunicaciones. Monográfico TICs en las aulas. Elementos Didácticos para la enseñanza, (242), 56-57.

Chavarría, L. B. (2016). Propuesta de un sistema de evaluación docente para la Facultad de Educación. Revista Educación, 16(2), 73-80.

Dávila, S. (2000). El aprendizaje significativo: esa extraña expresión utilizada por todos y comprendida por pocos. Contexto educativo, 9(7), 6-8.

Delgado, A.M., \& Oliver, R. (2006). La evaluación continua en un nuevo escenario docente. Revista de Universidad y Sociedad del Conocimiento, 3-1, 1-13.

Fernández March, A. (2006). Metodologías activas para la formación de competencias. Educatio siglo XXI, 24, 35-56.

Fernández, R. \& Pérez, C. A. (2013). ¿Mejoran las simulaciones en los laboratorios de química el aprendizaje de los alumnos? Percepciones de alumnos universitarios de primer curso de Química General. Revista Eureka sobre enseñanza y divulgación de las ciencias, 10(1), 47-65.

Fernández, R. (2014). Incorporación de simulaciones en el laboratorio de química general: influencia en el dominio afectivo del aprendizaje. Didáctica de las Ciencias Experimentales y Sociales, (28), 197-219.

Freeman, S., Eddy, S. L., McDonough, M., Smith, M. K., Okoroafor, N., Jordt, H., \& Wenderoth, M. P. (2014). Active learning increases student performance in science, engineering, and mathematics. Proceedings of the National Academy of Sciences, 111(23), 8410-8415.

Gómez, F. (2014). Educational innovation through ICTs in the university setting. what do students think of these practices? [La innovación educativa en la universidad a través de las TIC. ¿Qué ven los alumnos con estas prácticas?] RUSC Universities and Knowledge Society Journal,11(1), 49-60. doi:10.7238/rusc.v11i1.1657 
Guerra, S., González, N., \& García, R. (2010). Study on the use of ICT as teaching tools by university instructors. [Utilización de las TIC por el profesorado universitario como recurso didáctico] Comunicar, 17(35), 141-148. doi:10.3916/C35-2010-03-07

Ion, G., Barrera-Corominas, A., \& Tomàs-Folch, M. (2016). Written peer-feedback to enhance students' current and future learning. International Journal of Educational Technology in Higher Education, 13(1) doi:10.1186/s41239-016-0017-y

Lantz, M. E. (2010). The use of 'clickers' in the classroom: Teaching innovation or merely an amusing novelty?. Computers in Human Behavior, 26(4), 556-561.

López-Quintero, J.L., Varo-Martínez, M., Laguna-Luna A.M., \& Pontes-Pedrajas, A. (2016). Opinions on "Classroom Response System” by first-year engineering students. Procedia-Social and Behavioral Sciences, 228, 183-189.

Manzano-Agugliario, F. (2012). Propuesta de actividades y metodologías específicas para la mejora del bilingüismo en ingeniería. Universidad Almería.

Manzano-Agugliaro, F., Castro-García, M., Pérez-Romero, A. M., García-Cruz, A., Novas, N., \& SalmerónManzano, E. (2016). Alternative methods for teaching cadastre and remote sensing. Survey Review, 48 (351), 450-459

Manzano-Agugliaro, F., Salmerón-Manzano, E., \& Perea-Moreno, A. J. (2016). Las competencias transversales de las universidades del campus de excelencia agroalimentario (CEI-A3). Espiral. Cuadernos del Profesorado, 9(19), 68-77.

Pérez-Romero, A. M., Castro-jarcia, M., León-Bonillo, M. J., \& Manzano-Agugliaro, F. (2016). Learning effectiveness of virtual environments for 3D terrain interpretation and data acquisition. Survey Review, $1-10$.

Stowell, J. R., \& Nelson, J. M. (2007). Benefits of electronic audience response systems on student participation, learning, and emotion. Teaching of Psychology, 34, 253-258.

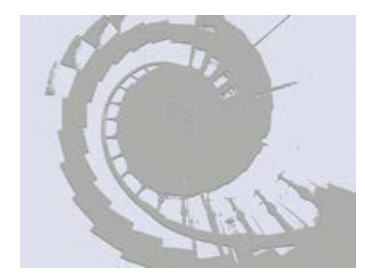

